\title{
DIPLOMACIA CULTURAL EN LA VEREZUELA DEL CHAVISMO: PINCELADAS DESDE LA POLITIZACIÓn
}

\section{Venezuelan cultural diplomacy during chavism}

\author{
Iván Alonso \\ Universidad de Sevilla \\ E-mail: ivan.alonso@orgc.csic.es \\ 0 Autor
}

Se presenta una aproximación para conocer las acciones culturales en el exterior del gobierno venezolano y su relación con las políticas culturales internas desarrolladas en el país desde 1999. Los documentos oficiales dejan ver que estas políticas han estado orientadas sobre todo a reivindicar los llamados "pueblos originarios", la vida de las comunidades, el recuerdo de la historia nacional y el apoyo político a la ideología gobernante. Algunas de estas acciones podrían no ayudar a los objetivos que establece la teoría sobre la diplomacia cultural.

Chávez; chavismo; diplomacia cultural; planificación; política cultural; politización; pueblos; Venezuela.

Chávez; chavism; cultural diplomacy; planning; cultural policy, politicization; nations; Venezuela.

Q Key words

This article is an approach to understand the external cultural actions of the Venezuelan government and its connections with the internal cultural policies that the country has developed since 1999. Official documents show that these policies have been mostly oriented to defend the "native nations", the community life and national history remembrance, and to support the ruling political ideology. Some of the actions may not be helpful when it comes to attaining the objectives set by cultural diplomacy theory. 


\section{Introducción}

Antes de comentar algunos aspectos de la acción cultural en el exterior desarrollada por el actual gobierno venezolano, conviene hacer al menos un brevísimo recorrido por sus políticas culturales. Tomamos como punto de partida un dato en el cual coinciden varios especialistas venezolanos que han estudiado las estadísticas económicas en torno a la cultura desde mediados del pasado siglo XX hasta el tiempo presente: los diferentes períodos presidenciales chavistas, con el propio Hugo Chávez a la cabeza desde 1999 hasta el 2013, y luego de su muerte, con el relevo de Nicolás Maduro hasta la actualidad, no han disminuido la inversión estatal en cultura en comparación con los gobiernos anteriores.

Incluso, como sugiere Gisela Kozak Rovero (2015), se podría afirmar que en estos casi 18 años de la llamada "Revolución Bolivariana" se ha aumentado la inversión estatal en cultura, si se toma en cuenta que aparte del presupuesto ordinario, se han hecho transferencias al sector a través de otras vías como los créditos adicionales, las erogaciones especiales para proyectos específicos o las aportaciones económicas de otros ministerios diferentes al de Cultura (p. 43). Al respecto de esta continuidad, Carlos Enrique Guzmán Cárdenas (2013), sociólogo de referencia en Venezuela en las investigaciones sobre Economía de la Cultura y Consumo Cultural, expone lo siguiente:

El crecimiento institucional de la inversión pública cultural consolidada desde 1965, cuando empezó a funcionar el INCIBA, hasta la formulación del presupuesto para el ejercicio fiscal 2010 [...] correspondiente al actual Ministerio del Poder Popular para la Cultura, ha estado signado por una progresión nominal tendencial cercana al 0,53\% del total del presupuesto nacional (p. 249).

A grandes rasgos, lo que viene a explicar Guzmán Cárdenas es que desde 1965, cuando se creó el Instituto Nacional de Cultura y Bellas Artes (INCIBA), primera entidad pública encargada de la gestión de los proyectos culturales en Venezuela, hasta el tiempo presente, la inversión del Estado en cultura ha estado en torno al medio punto porcentual del presupuesto nacional. El INCIBA se transformó luego, en 1975, en el Consejo Nacional de la Cultura (CONAC), entidad adscrita a la Secretaría de la Presidencia de la República, hasta que en el año 2005, durante el segundo período de Hugo Chávez, desaparece el CONAC y en su lugar se crea el Ministerio del Poder Popular para la Cultura, teniendo como primer titular a Francisco de Asís Sesto Novás, mejor conocido como Farruco, arquitecto y profesor universitario que desde su juventud había militado en organizaciones políticas de izquierda, y que había desempeñado diferentes cargos públicos desde el inicio de la administración chavista en 1999.

Bien pareciera que la financiación como tal no ha sido un problema para la cultura en la era del chavismo. Conviene entonces fijar la mirada en la calidad e impacto de esa inversión. En otras palabras, evaluar cuáles objetivos se han logrado o no en materia cultural a través de esas transferencias de presupuesto. Llegado a este punto recogemos una queja que comparten los dos autores antes mencionados, y es la ausencia en la actualidad de estadísticas oficiales totales, completas y normalizadas que puedan dar una idea del impacto del consumo cultural y la eficacia de las políticas puestas en marcha. A pesar de esta ausencia, los especialistas han ido tomando para sus investigaciones algunos estudios estadísticos alternativos realizados por instituciones académicas y culturales sobre determinados sectores de la industria cultural.

De esa forma, Kozak Rovero (2015) aborda en una parte de su estudio el sector editorial y la lectura utilizando como fuente una encuesta realizada en el año 2012 por el Centro Nacional

\section{Los diferentes períodos presidenciales chavistas no han disminuido la inversión estatal en cultura en comparación con los gobiernos anteriores}


del Libro (CENAL), y las investigaciones "Venezolanos comelibros", de Lisseth Boon y Jesús Alberto Yajure, y "El libro: entre cuentos y cuentas", de Carlos Delgado Flores, ambas realizadas en el año 2013 (p. 45). La autora aporta datos que demostrarían que durante las administraciones chavistas se ha incrementado de forma significativa la producción de libros, a precios prácticamente simbólicos, gracias a iniciativas estatales como la creación de la editorial pública "El Perro y la Rana", que según los datos expuestos en su propia página web, en sus diez años de existencia ha publicado 4500 títulos. "Venezuela ha triplicado el número de títulos publicados en el país por habitante: de 4 títulos al año en 2004 ha pasado a 12 en el año 2012" (p. 45).

No obstante, estas cifras, en principio optimistas, tienen su contraparte en las propias estadísticas citadas por Kosak Rovero (2015). Por ejemplo, según la encuesta del CENAL, el 80\% de los entrevistados afirma no haber obtenido nunca un libro publicado por el Estado (p. 45). Asimismo, las obras literarias señaladas como más leídas por los entrevistados fueron dos clásicos de la Literatura venezolana, Doña Bárbara de Rómulo Gallegos y Casas Muertas de Miguel Otero Silva, que ya estaban posicionadas entre las favoritas del público en las encuestas previas al advenimiento de la Revolución bolivariana. Para la autora, "la intervención del Estado no ha modificado sustancialmente los hábitos mayoritarios de lectura anteriores a 1998” (p. 46).

Ante estos números, cabe plantear la siguiente pregunta: si las administraciones chavistas han invertido tal cantidad de fondos que han permitido en pocos años triplicar la producción de libros, y además a precios simbólicos, ¿por qué ese esfuerzo económico no ha tenido un impacto proporcional en la ciudadanía? La autora atisba una respuesta:

[...] el gobierno edita de manera caprichosa y con marcado sesgo ideológico, lo cual desde luego aleja a públicos no alineados. Este alejamiento tiene otra arista importante: la competencia con el sector privado es absolutamente desleal en detrimento del conocimiento de la obra de autores no oficialistas por los precios de los libros no subsidiados. Por ejemplo, no existe un programa de compras para bibliotecas que asegure la presencia de la diversidad editorial venezolana en todo el país; los autores no identificados con la revolución no cuentan pues con el apoyo del estado y sus recursos, que pertenecen a toda la población, para el conocimiento de su trabajo y la búsqueda de potenciales lectores (p. 46).

Ciertamente, los gobiernos chavistas han publicado muchos libros, pero pareciera que la lógica empleada no ha sido tanto el fomento de una idea amplia, plural y diversificada del conocimiento, sino la consolidación de los lineamientos ideológicos y políticos de la Revolución bolivariana, y el enaltecimiento de los valores que el chavismo ha declarado como convenientes y apropiados: el recuerdo idealizado de los "padres de la patria" o héroes de la gesta independentista del siglo XIX, la puesta en valor del acervo indígena y negro-africano, el rescate de lo tradicional y telúrico, el afianzamiento de un espíritu latinoamericanista, la promoción de la cultura comunal, entre otras. Podríamos hablar de un cierto tono nacionalista y etnocentrista en esta política editorial del Estado; o la utilización de la cultura como arma en la lucha contra el cosmopolitismo y la noción de "bellas artes" y "alta cultura" asociada a las élites opositoras.

Una situación similar describe Libia Villazana (2008) en su trabajo sobre el sector audiovisual cuando analiza la experiencia de uno de los proyectos culturales bandera de la administración bolivariana, la "Villa del Cine". Esta infraestructura inaugurada en junio de 2006 cuenta "con un espacio de cuatro hectáreas de extensión, así como un estudio dedicado a la producción de cine y otro a la televisión que permiten una capacidad de producción cinematográfica de hasta

\section{La lógica} empleada no ha sido tanto el fomento de una idea plural del conocimiento, sino la consolidación de los lineamientos ideológicos y políticos de la Revolución bolivariana 
12 películas al año” (p. 165). Para el momento en que Villazana realiza su trabajo, en la Villa del Cine ya se habían producido 200 documentales que, al igual que con los datos antes señalados para la industria editorial, representa un número significativo. No obstante, todos estos documentales habían sido realizados por grupos comunitarios que narraban su vida cotidiana. Asimismo, comenta la autora que entre las producciones que ya se habían rodado o las que contarían con apoyo financiero en el futuro, se contaban obras sobre los próceres patrios como Francisco de Miranda, Ezequiel Zamora y el propio Simón Bolívar, entre otros. Como se puede ver, se repite en la industria audiovisual estatal la intención de fortalecer el programa ideológico del gobierno en una actitud casi propagandística. Apunta Villazana:

Contar con un complejo audiovisual con tecnología avanzada, como es el caso de esta Villa, es el sueño de todo país latinoamericano, sobre todo de aquellos países con industrias audiovisuales más frágiles. Venezuela ha logrado edificar y poner en funcionamiento inmediato ese sueño. Sin embargo, utilizar estas instalaciones para producir proyectos audiovisuales dirigidos mayoritariamente al fortalecimiento de lo estipulado en la Constitución Nacional equivale a cegar al proyector de cine e incluso puede equivaler paradójicamente a alimentar el desencantamiento del público venezolano por su cine. Pero las líneas editoriales parecen apuntar hacia esta dirección, así lo expresa la directora del complejo, Lorena Almarza: "Nosotros, estamos haciendo guiones que reflejan justicia, solidaridad, es decir, los valores que expresa la Constitución de la República Bolivariana de Venezuela, los valores fundamentales que nos permitirán construir ese nuevo ciudadano".

Villazana sugiere la posibilidad de un público desencantado con un cine eminentemente partidista, al igual que Kosak Rovero apunta a una proporción de lectores no alineados que no se sienten atraídos hacia publicaciones que destacan unas determinadas ideas políticas. Se encuentra en esta arista la paradoja de unas políticas que pretendiendo incluir lo que presuntamente había sido excluido en el pasado -lo popular, lo telúrico, lo nacional- terminan siendo excluyentes.

\section{La cultura como sustancia del proyecto político}

Cuando se revisan algunos de los documentos oficiales de planificación elaborados por la administración chavista, se puede observar que no se esconde ni se maquilla con eufemismos la intención de utilizar a la cultura para afianzar entre la población el ideario de la Revolución bolivariana. Tomando prestado el juego de palabras que hace Villazana en el título de su investigación, estaríamos hablando de una "política cultural politizada".

Como apuntan Emilia Bermúdez y Natalia Sánchez (2009), esta posición beligerante de las políticas culturales en la administración chavista se acentuó a partir de abril de 2002, fecha en la cual se produjo el fallido golpe de Estado que mantuvo a Hugo Chávez durante dos días fuera del poder. Recuerdan las autoras que en ese momento se operó un cambió semántico significativo en la dialéctica oficial; ya no se habló tanto de construir la Revolución bolivariana, sino que empieza a aparecer con más fuerza la idea del "Socialismo del siglo XXI"; y se insiste en consignas nacionalistas y populistas que claman por el rescate de la venezolanidad, de la memoria y de las tradiciones (p. 561). Como una ofensiva para marcar el territorio ante la amenaza

\section{Esta posición}

beligerante de las

politicas culturales

se acentuó a partir

de abril de 2002,

fecha en la cual se

produjo el fallido

golpe de Estado

que mantuvo a

Hugo Chávez

durante dos días

fuera del poder. 
de las fuerzas opositoras, el gobierno muestra, ya sin cortapisas, su objetivo real de construir un Estado socialista.

La elaboración de "planes de la nación” plurianuales, como instrumento de planificación para establecer metas de desarrollo de largo aliento y administrar los recursos, se convirtió en una práctica común de los gobiernos democráticos venezolanos que vinieron luego de la caída de la dictadura de Marcos Pérez Jiménez en 1958; de hecho, ese mismo año se creó la Oficina Central de Coordinación y Planificación. El Primer Plan de la Nación se elaboró para el período 1960 - 1964, durante el gobierno de Rómulo Betancourt, candidato del partido socialdemócrata Acción Democrática (AD). En el momento de la llegada al poder de Hugo Chávez, tras las elecciones presidenciales de diciembre de 1998, regía el Noveno Plan de la Nación para el período 1995 - 1999, implantado durante el gobierno de coalición del ex socialcristiano Rafael Caldera.

Los gobiernos chavistas han mantenido la práctica de elaborar estos programas de planificación del desarrollo, que incluso están contemplados como una obligación del Poder Ejecutivo en la Constitución de la República Bolivariana de Venezuela (1999). Gradualmente desde el gobierno se le ha ido imprimiendo una impronta ideológica a un instrumento que debería tener más un carácter técnico. De esa forma, el primer documento de planificación de la administración chavista aún mantuvo un nombre similar a la tradición que se había instituido desde 1958, y se denominó "Plan de Desarrollo Económico y Social de la Nación, 2001-2007"; pero el siguiente ya adoptó un nombre más comprometido: "Proyecto Nacional Simón Bolívar; Primer Plan Socialista de Desarrollo Económico y Social de la Nación, 2007-2013”. Resulta significativo que sólo con observar su nombre se puede detectar dos alusiones claras a lo que podríamos llamar la "cultura del chavismo"; por una parte, la referencia a un pasado heroico fundamentado en la gesta independentista con la inclusión de "Simón Bolívar" como imagen omnipresente; y por otra parte, la declaración directa del socialismo como doctrina política de cabecera. Actualmente en la administración venezolana rige el "Plan de la Patria, Segundo Plan Socialista de Desarrollo Económico y Social de la Nación, 2013 - 2019”. Ya no aparece Simón Bolívar en la denominación, pero en su lugar se alude a la patria, una referencia igualmente de tono nacionalista.

Este último documento de planificación básicamente contiene un listado amplio de "objetivos nacionales" que el gobierno pretende cumplir en el período señalado. Cada uno de estos objetivos luego se desarrolla con otro listado de "objetivos estratégicos y generales". Se echa de menos información más técnica de cómo se planean conseguir cada uno de los objetivos, para que así el documento sea algo más que una relación de buenas intenciones. Pero volviendo al tema de las políticas culturales, vale la pena citar un fragmento del Plan de la Patria para comprender la orientación de la administración chavista sobre el tema. Dentro del objetivo nacional "Construir una sociedad igualitaria y justa", se desarrolla el objetivo estratégico "Potenciar las expresiones culturales liberadoras del pueblo", con los siguientes postulados:

1) Incrementar sostenidamente la producción y distribución de bienes culturales a nivel nacional. 2) Fortalecer las editoriales que incluyan espacios de participación del poder popular en la política editorial mediante la generación de imprentas regionales. 3) Aumentar los espacios y la infraestructura cultural a disposición del pueblo, que permitan el desarrollo local de las artes. 4) Impulsar y ampliar la red de intelectuales, artistas, cultores y cultoras, y la organización de redes comunitarias culturales. 5) Desarrollar

\section{Gradualmente desde el gobiemo se le ha ido imprimiendo una impronta ideológica a un instrumento que debería tener} más un carácter técnico 
investigaciones sobre las tradiciones culturales que impulsen el conocimiento y práctica cultural. 6) Visibilizar la identidad histórico-comunitaria en conexión con la Misión Cultura Corazón Adentro. 7) Consolidar el protagonismo popular en las manifestaciones culturales y deportivas, centrado en la creación de una conciencia generadora de transformaciones para la construcción del socialismo. (Plan de la Patria, 2013, 4 de diciembre, p. 22).

Si bien hay objetivos que pueden resultar en principio loables, como por ejemplo incrementar la producción de bienes culturales o fomentar la instalación de imprentas, en otros de ellos, sin entrar a valorar su calidad o adecuación, se aprecia el giro ideológico del cual hemos venido hablando: primacía de lo comunitario y popular, exaltación de las tradiciones nacionales en relación con la Historia, y fomento del socialismo. Se trata sólo de un fragmento del Plan, pero en una revisión exhaustiva, que excede al objetivo de este trabajo, se pueden encontrar más huellas de esta intencionalidad ideológica que estaría rozando el adoctrinamiento cultural.

Max Römer Pieretti (2014) apunta que la identidad política que ha construido el chavismo se empezó a fraguar a través de "la dialéctica que generó Chávez desde el mismo momento de su juramento como presidente, al determinar una diferencia entre el pasado y el futuro para dar pie a su proyecto" (pp. 50-51). En otras palabras, el chavismo ha difundido en su discurso dos categorías; por una parte, la que perciben como propia, nueva y revolucionaria, donde culturalmente ubican el acervo indígena, negro-africano, las tradiciones, el folclore y la vida popular y comunitaria. Políticamente, a esta categoría corresponde un enaltecimiento idolátrico de los héroes de la Guerra de la Independencia del siglo XIX y una suerte de reinterpretación del socialismo. La otra categoría, la que el chavismo ha decidido que corresponde al contrario, la de los que defienden los valores anteriores a 1999: la IV República, está culturalmente vinculada a lo blanco-europeo, la herencia española y occidental, las élites conocedoras y cultivadoras de las bellas artes y la alta cultura. Por supuesto, políticamente a esta categoría "contrarrevolucionaria” corresponde una defensa de la democracia burguesa capitalista.

La identidad venezolana después de la irrupción de Chávez pretende por una parte excluir al que de alguna manera fue dominante en el pasado. Blanco, rico, político, cercano a otras culturas, especialmente aquellas que pertenecen a los hemisferios norte y occidental. Por la otra, destacar la originalidad del pueblo base, del amerindio, de su fusión con el negro oprimido (Römer Pieretti, 2014, p. 62).

Esta postura dicotómica excluye la posibilidad de políticas culturales que apuesten por el mestizaje, la integración y la pluralidad de orígenes que conforman la identidad venezolana. Se pretende crear una suerte de “purismo” venezolano que se escapa a cualquier lógica.

\section{De la cultura politizada a la diplomacia cultural}

Realizando una lectura rápida de la agenda cultural de la Embajada de Venezuela en España, se puede constatar que en buena medida los mismos valores, objetivos y lineamientos ideológicos presentes en las políticas culturales desarrolladas al interior de la República, traspasan las fronteras y permean la acción exterior. Por ejemplo, para el momento de redactar este trabajo, la noticia más reciente en la sección cultural de la página web de la embajada de Venezuela en Madrid se titula "Vigencia del pensamiento libertario e integracionista de Francisco de Miranda recorrió ciudades de Europa”. La nota narra la presentación, auspiciada por la Embajada, del
El chavismo ha difundido en su discurso dos categorías: la que perciben como propia, nueva y revolucionaria y la que el chavismo asigna al contrario, la de los valores anteriores a 1999 
libro Francisco de Miranda, precursor de las independencias de América Latina, obra de la historiadora Carmen Bohórquez, llevada a cabo el 15 de noviembre de 2016 en la Casa de América de Madrid. En una parte de la noticia se explica lo siguiente:

El embajador Isea, en las palabras de bienvenida destacó que esta edición del libro realizada bajo el patrocinio de la Embajada, contribuirá a que en Europa "tomen conciencia del aporte que Miranda hizo en la construcción del nuevo pensamiento y el impacto real que tuvo su presencia en acontecimientos revolucionarios que hicieron historia en el siglo XIX”, dijo al tiempo que subrayó el fervor y la rigurosidad de la autora en esta investigación (Embajada de Venezuela en España, 2016).

Otra de las actividades recientes que reseña la web de la legación diplomática es el foro "Los Derechos de los Pueblos Originarios: Una realidad en América Latina”, que se celebró el 20 de octubre de 2016 en el Centro de la Diversidad Cultural de Venezuela en España (CDCVE), instalación definida como el brazo cultural de la Embajada. La reseña explica de la siguiente forma los objetivos de la actividad cultural:

Mención especial merece la Constitución de la República Bolivariana de Venezuela (1999), que dedicó todo un capítulo al reconocimiento de las comunidades indígenas, sus organizaciones sociales, políticas, económicas y culturales, como sujetos de derecho; además, de propugnar el mantenimiento y desarrollo de su identidad étnica y cultural, entre otros, considerada como una de las primeras normas sancionadas sobre la materia a nivel mundial.

Esta actividad pretende convertirse en un espacio que redunde hacia una mayor sensibilización en relación al tema indigenista y está dirigida a funcionarios públicos, estudiantes universitarios, académicos; activistas, líderes y organizaciones de las comunidades indígenas, y público general (Embajada de Venezuela en España, 2016).

Entre la oferta cultural de esta Embajada en los últimos meses también figura la conferencia "El 1S y el camino de la Revolución Bolivariana", dictada por el propio embajador, Mario Isea Bohórquez, el 9 de septiembre de 2016. Según la información presentada por la embajada, la conferencia abordó el proceso de solicitud de referéndum revocatorio llevado a cabo por la oposición política al chavismo, que en opinión del diplomático, se trata de una nueva estratagema para desestabilizar el país:

Isea aseguró que el gobierno bolivariano "aprendió la lección del 11 de abril 2002 (fecha del golpe de Estado contra el presidente Hugo Chávez) y no permitiremos que el guión de la derecha vuelva a tener éxito, por eso, ante esta nueva arremetida se están tomando las medidas preventivas necesarias".

El diplomático realizó durante su intervención un repaso por las últimas dos décadas de la historia de Venezuela, en la que se evidencia cómo a través de los años los actores protagonistas de los hechos violentos de desestabilización y de intentos de golpes de Estado han sido los mismos.

"Personajes como Leopoldo López, Antonio Ledezma, María Corina Machado, Henrique Capriles y Yon Goicochea, y éste último aquí en España es presentado casi como un héroe, cuando acaba de ser detenido por tenencia de explosivos, y todos los demás han participado repetidamente desde 2002 en diversos episodios de violencia con el objetivo

\section{En la agenda} cultural de la Embajada de Venezuela en España, se puede constatar que los mismos valores, objetivos $y$ lineamientos ideológicos permean la acción exterior 
de derrocar al Gobierno y hacer desaparecer los logros de la Revolución Bolivariana”, explicó (Embajada de Venezuela en España, 2016).

La página web describe algunas otras actividades culturales como el concierto de Sandino Primera, cantante plenamente identificado con el chavismo e hijo del fallecido cantautor de protesta y militante del Partido Comunista de Venezuela, Alí Primera. También se describen funciones de cine venezolano organizado por el CDCVE, con una programación dedicada a los diferentes pueblos indígenas, entre otras. Excedería al alcance de este trabajo describir de forma pormenorizada toda la agenda cultural de la representación diplomática venezolana en Madrid; pero al menos con las anteriores actividades señaladas se deja entrever que la acción cultural de Venezuela en el exterior sigue similares, por no decir idénticos, lineamientos de las políticas culturales internas: exaltación de los llamados pueblos originarios, recuerdo idolátrico de los próceres de la Guerra de Independencia y defensa de las causas e ideologías políticas del gobierno.

Recuerda Edgar Montiel (2010) que tras los ataques terroristas del 11 de septiembre de 2001, desde muchos ámbitos se empezó a buscar la explicación de lo ocurrido en la cultura, más allá de la política o la economía. Quizás como nunca se puso en evidencia el choque simbólico significativo y la ausencia de comunicación y comprensión entre las diversas formas de entender el mundo por parte de las diferentes culturas. Más de 15 años después de lo ocurrido en Nueva York, sigue presente, y con más vigencia que nunca, el malestar cultural, con el yihadismo y todas sus consecuencias como su manifestación más clara.

A grandes rasgos es ésta la razón por la cual en los últimos años los estudiosos y profesionales de las relaciones internacionales han dirigido cada vez más su mirada a la cultura y al poder simbólico, teniendo al estadounidense Joseph Nye y su teoría del Soft Power como punto de referencia de la Diplomacia Cultural. Pero la cultura no es un aspecto nuevo de atención para la diplomacia, "ha estado siempre presente en la agenda gubernamental de la política exterior, y fue reconocida como un "tercer pilar", junto a la política (seguridad) y al comercio (economía), en las relaciones entre estados después de las dos guerras mundiales" (Said Saddiki, 2009, p. 108). No obstante, el nuevo escenario geopolítico, con los choques entre Occidente y Oriente en la primera línea de actualidad, obliga a reforzar el entendimiento y el diálogo entre culturas.

(...) la diplomacia pública y la diplomacia cultural están relacionadas con el denominado "poder blando" (soft power), un concepto introducido por Joseph Nye, el cual define como "la habilidad para conseguir lo que uno pretende por medio de la seducción, y no por medio de la coerción o el pago. Surge del carácter atractivo que tienen la cultura, la política o los ideales políticos de un país. Cuando nuestra política es vista como legítima a ojos de los demás, nuestro poder blando se incrementa mucho” (Said Saddiki, 2009, p. 109).

Refrescamos estos conceptos sobre poder blando y diplomacia cultural, que son ampliamente conocidos por la crítica, con la intención de reflexionar en torno a las actividades culturales promovidas por la diplomacia venezolana. Si las acciones culturales en las relaciones internacionales deben tener como objetivo abrir las puertas al diálogo a través de contenidos seductores que creen un clima favorable para causar impacto y empatía en los otros, pues poco puede servir para esta causa una programación nacionalista, etnocéntrica, partidista y en definitiva

\section{En los últimos años los estudiosos y profesionales de las relaciones internacionales han dingido cada vez más su mirada a la cultura y al poder simbólico}


excesivamente politizada, que pone su énfasis en la reivindicación antes que en la conciliación; en las diferencias antes que en los puntos en común ${ }^{1}$.

Saddiki (2009), citando a su vez a Von Bismarck, recuerda que la "La diplomacia es el arte de hacer amigos en el extranjero" (p. 110), y sugiere demás que es un error el que comenten algunos autores al confundir a la diplomacia cultural con la propaganda. Si partimos de las actividades antes descritas de la Embajada de Venezuela en Madrid, se pudiera interpretar que sus acciones culturales están únicamente dirigidas a sus "cofrades", es decir, aquellos sectores y grupos de la sociedad española que de antemano comparten su ideario político; una diplomacia cultural "para los amigos" y no "para hacer nuevos amigos". Se trataría de una lógica que en principio contradice los objetivos que los expertos señalan como propios de la diplomacia cultural: influir positivamente en la opinión pública de un Estado extranjero, crear bases de confianza, promover el entendimiento mutuo y elevar el prestigio del país, entre otros.

Una actitud menos beligerante y apoyada en contenidos más universales es la que realiza la Embajada de Venezuela ante el Reino Unido, ubicada en la ciudad de Londres. Esta legación diplomática centra su agenda cultural en una instalación, la "Casa de Miranda”, que contiene a su vez el "Bolívar Hall", una amplia sala adaptable que se puede utilizar para conciertos, presentaciones y exposiciones. En una revisión rápida a la programación cultural de legación diplomática se puede constatar que abundan los conciertos musicales, sobre todo de intérpretes y agrupaciones que cultivan géneros tradicionales del folclore venezolano. Sirva de ejemplo el siguiente fragmento de la nota que anuncia el inicio de la programación de otoño del 2016 tras una nutrida oferta estival:

La programación de verano culminó el pasado 18 de agosto con el concierto del Maestro Omar Acosta, reconocido compositor y flautista, en conmemoración del Bicentenario del Fallecimiento del Generalísimo Francisco de Miranda. Acosta estuvo acompañado en el escenario por Nuria Cazorla, bailaora de flamenco y el conocido virtuoso del cuatro, Leo Rondón. El repertorio incluyó las composiciones Natalia, (vals de Antonio Lauro); Canto de ordeño (Antonio Estévez); Atardecer (vals de Luis Laguna y Lencho Amaro); La Comparsa (danzón cubano de Ernesto Lacuna); Por una cabeza (tango de Carlos Gardel); Preludio No 1 (J.S. Bach) y El diablo suelto (vals joropo de Heraclio Fernández). Otras piezas que del programa fueron Solo de pajarillo (Joropo); Vals de Lucía; Pasaje; Carolina (vals) y El cucarachero (merengue venezolano), todas autoría de Acosta. La presentación cosechó un éxito notable, con un público desbordante compuesto por miembros de la academia musical clásica de Londres, especialmente del Instituto Britá-

1 Por ejemplo, bien es sabido que el 12 de octubre se celebra en España la "Fiesta Nacional", y esa es la denominación que recibe esta fecha en el ordenamiento jurídico español. En ese sentido, que desde la Embajada venezolana en Madrid se lleve a cabo en días cercanos a la efeméride un foro reivindicativo de los derechos de los pueblos indígenas con la justificación de que el 12 octubre es el "Día de la Resistencia Indígena”, podría interpretarse como una actitud poco amistosa o seductora que no estimula el diálogo, sino que pone el dedo en la llaga. No queremos con este ejemplo sugerir que las autoridades diplomáticas venezolanas deban renunciar a hacer cualquier tipo de reivindicación o protesta que su gobierno considere oportuna y necesaria, sino que la elemental lógica invita a pensar que la diplomacia está para limar asperezas y sólo levantar la voz como último recurso, cuando el devenir de los hechos no deja otra alternativa, y no por antiguas polémicas.

2 La "Casa de la Miranda" es la vivienda que ocupó Francisco de Miranda (1750-1816), prócer de de la Independencia de Venezuela, junto a su familia durante la época en que vivió en Londres entre 1803 y 1810. En 1978 el gobierno venezolano adquirió la Casa Mirada y los edificios adyacentes donde se construyó el complejo cultural que alberga el Bolívar Hall.

\section{Se trataría de} una lógica que en principio contradice los objetivos que los expertos señalan como propios de la diplomacia cultural 
nico de Flauta, que reaccionó con expresividad a los ritmos y melodías que evocaron el paisaje cultural, musical, y hasta geográfico, de Venezuela. (Embajada de Venezuela en Reino Unido, 2016).

Si bien es cierto que hay un predominio del sustrato telúrico-folclórico ensalzado en las políticas culturales de los gobiernos chavistas, también es cierto que la música tradicional, a través de la mirada de la diplomacia cultural, resulta más atractiva y empática que las conferencias y manifestaciones donde únicamente se defiende una determinada parcialidad política. A diferencia de lo que ocurre con la programación cultural de la Embajada en Madrid, cualquier persona que asista como espectador a una de estas representaciones de música tradicional en la Casa de Miranda, independientemente de que comparta o no los lineamientos ideológicos del chavismo, puede sentirse cautivado por el encantamiento de la música y despertar así una actitud positiva hacia Venezuela.

Con los casos específicos revisados se puede apuntar como conclusión que se extraña mayor pluralidad y diversidad en la diplomacia cultural del gobierno venezolano. No se puede juzgar la diplomacia cultural de un país únicamente por las actividades que promueven algunas de sus representaciones en el exterior, pero pueden servir como pistas para construir su caracterización. En ese sentido, sería necesario investigaciones más amplias que analizaran la participación oficial de Venezuela en foros y organizaciones internacionales, proyectos de integración, redes y medios de comunicación con alcance en el exterior, ferias, festivales, reuniones y congresos académicos, entre muchos otros eventos. No obstante, esta primera aproximación sugiere que en su actuación cultural la diplomacia venezolana estaría orientada de forma similar a la política cultural interior, con un claro énfasis en la difusión de valores nacionalistas y un evidente alineamiento con la ideología política gobernante. Se trata así de una estrategia que a nuestro juicio no ayuda suficientemente a la construcción de puentes para el diálogo en un mundo decididamente multipolar.

\section{Bibliografía}

Bermúdez, E., \& Sánchez, N. (2009). Política, cultura, políticas culturales y consumo cultural en Venezuela. Espacio abierto, $18(3)$.

Embajada de la República Bolivariana de Venezuela en España (2016, 14 de septiembre). [Foro en Madrid] El diálogo es el camino que propone la Revolución para que la oposición abandone la violencia. Recuperado de http://embajadadevenezuela.es/eventos-culturales/6043-eldialogo-es-el-camino-que-propone-la-revolucion-para-que-la-oposicion-abandone-laviolencia

Embajada de la República Bolivariana de Venezuela en España (2016, 7 de octubre). Venezuela difunde en Europa reivindicaciones de los pueblos originarios de América Latina. Recuperado de http://embajadadevenezuela.es/eventos-culturales/6070-venezuela-difunde-eneuropa-reivindicaciones-de-los-pueblos-originarios-de-america-latina

Embajada de la República Bolivariana de Venezuela en España (2016, 23 de noviembre). Vigencia del pensamiento libertario e integracionista de Francisco de Miranda recorrió ciudades de Europa. Recuperado de http://embajadadevenezuela.es/eventos-culturales/6156vigencia-del-pensamiento-libertario-e-integracionista-de-francisco-de-miranda-recorriociudades-de-europa 
Embajada de la República Bolivariana de Venezuela en el Reino Unido (2016, 9 de septiembre). Tras exitosa temporada estival, el Bolivar Hall se prepara para iniciar su programación de otoño en Londres. Recuperado de http://reinounido.embajada.gob.ve/index. php?option $=$ com_content $\&$ view $=$ article $\&$ id $=361 \% 3$ Aexitosatemporada $\&$ catid $=20 \% 3 \mathrm{~A}$ eventos\&Itemid $=15 \&$ lang $=$ es

Guzmán Cárdenas, C. E. (2014). Economía y Política Cultural en Venezuela. Revisión y Perspectivas. Anuario ININCO/Investigaciones de la Comunicación, 25(1), 225-270.

Kosak Rovero, G. (2014). Revolución Bolivariana: políticas culturales en la Venezuela Socialista de Hugo Chávez (1999-2013). Cuadernos de Literatura, 19(37), 38-56.

Montiel, E. (2010). Diplomacia cultural. Un enfoque estratégico de Política Exterior para la era intercultural. Cuadernos UNESCO Guatemala, 2, 1-26.

Poder Legislativo de Venezuela (2013, 4 de diciembre). Plan de la Patria. Segundo Plan Socialista de Desarrollo Económico y Social de la Nación 2013-2019. Gaceta Oficial de la República Bolivariana de Venezuela, (6.118).

Römer Pieretti, M. (2014). Venezuela a partir de Chávez: identidad cultural y política. Historia y Comunicación Social, 19, 55-65.

Saddiki, S. (2009). El papel de la diplomacia cultural en las relaciones internacionales. Revista CIDOB d'afers internacionals, 88, 107-118.

Villazana, L. (2008). De una política cultural a una cultura politizada: La República Bolivariana de Venezuela y su revolución cultural en el sector audiovisual. E pluribus unum, 161-73. 